Instituto Internacional de Investigación y Desarrollo Tecnológico Educativo INDTEC, C.A.

DOI: https://doi.org/10.29394/Scientific.issn.2542-2987.2019.4.11.11.218-238

OAI-PMH: http://www.indteca.com/ojs/index.php/Revista Scientific/oai

Artículo Original / Original Article

\title{
El Agnosticismo Pedagógico de la Educación Andragógica de los Derechos Humanos
}

Autor: Richard Manuel Salazar Brito Universidad Bicentenaria de Aragua, UBA raam2623@hotmail.com

Turmero, Venezuela https://orcid.org/0000-0002-9986-4145

\section{Resumen}

La presente investigación está circunscrita bajo un enfoque epistemológico interpretativo, orientado a la comprensión conceptual de los derechos humanos desde la perspectiva crítica del porqué del agnosticismo pedagógico en la educación andragógica. Tiene como objetivo general, analizar la evolución histórica de los derechos humanos en la República Bolivariana de Venezuela, con el propósito de conocer el porqué del agnosticismo pedagógico de los derechos humanos en la ciudadanía. Para ello se realizó una revisión bibliográfica de las bases filosóficas de los derechos humanos, la evolución filosófica de las constituciones existentes en el país, desde la época denominada transición a la democracia, se indagar la fundamentación teórica y filosófica de los derechos humanos en Venezuela, como axioma formativo para la gnoseología andragógica. Como conclusión, se da a conocer el porqué del agnosticismo pedagógico de la educación andragógica de los derechos humanos.

Palabras clave: comprensión; educación; derechos humanos. 


\section{The Pedagogical Agnosticism of Andragical Education of Human Rights}

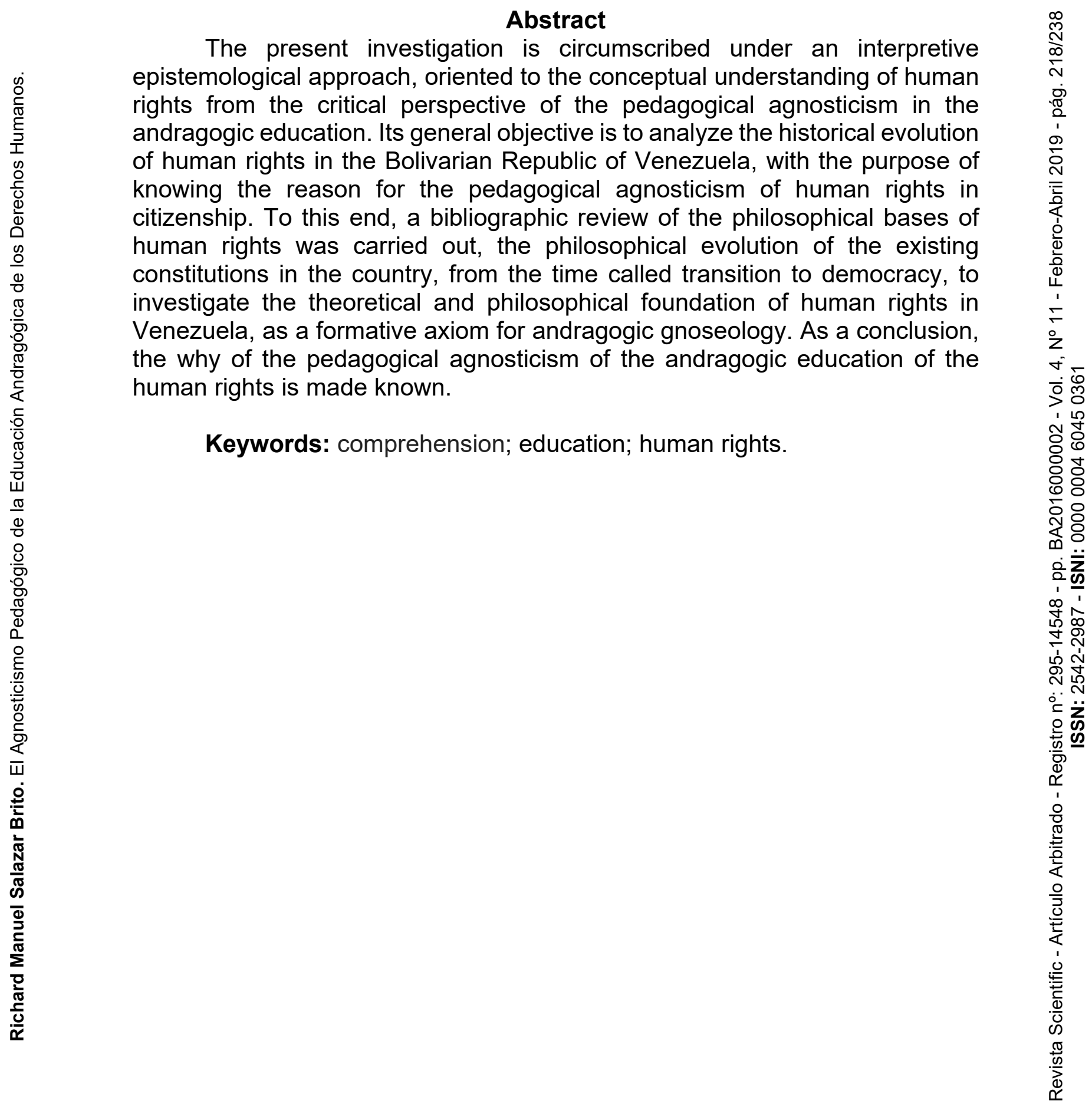

Date Received: $18-06-2018$

Date Acceptance: 30-09-2018 


\section{Introducción}

El hombre desde su origen ha tenido una interacción inmediata con el ambiente, en función a las necesidades e intereses de estos, sin embargo, dichas necesidad se han transformado de acuerdo con ciertos inconvenientes que han surgido con la evolución de la sociedad y la tecnología. Es por lo que, en el siglo $X X$, se han derivado diversos cambios e innovaciones quebrantando, de manera directa, la conducta del individuo, debilitamiento ambiental, el uso excesivo de los recursos naturales, entre otras causas. Esto causado, hoy en día, inquietud en el mundo, en función de sostener la vida en la tierra.

El objetivo de la presente investigación, es analizar la evolución histórica de los derechos humanos en la República Bolivariana de Venezuela, con el propósito de conocer el porqué del agnosticismo pedagógico de los derechos humanos en la ciudadanía.

Para comenzar con la caracterización del objeto de la Investigación, comenzaremos por definir que son los Derechos Humanos, entendiendo según La Oficina del Alto Comisionado para los Derechos Humanos (ACNUDH, 2012), como:

Los derechos humanos son derechos inherentes a todos los seres humanos, sin distinción alguna de nacionalidad, lugar de residencia, sexo, origen nacional o étnico, color, religión, lengua, o cualquier otra condición. Todos tenemos los mismos derechos humanos, sin discriminación alguna. Estos derechos son interrelacionados, interdependientes e indivisibles (pág. 1).

Ahora bien, existen muchas definiciones, a razón de que existen diversas concepciones sobre la decencia humana y los derechos humanos; Pero todos van dirigidos a protegen la dignidad humana, para la satisfacción de sus necesidades esenciales para vivir. Pero esa lucha por la afirmación de los derechos del ser humano, han evolucionado de acuerdo con las luchas políticas frente a los abusos de poder, que se fundamentó en las luchas 
reivindicativas de los sectores sociales excluidos o sometidos, en búsqueda de asegurar su dignidad y la equidad social.

Por lo antes expuesto, en la Declaración Universal de los Derechos Humanos en el artículo 26, el cual trata sobre el derecho a la educación, desde una perspectiva holística, enunciando que todos tienen derecho a la educación, en función al desarrollo del individuo, la tolerancia, la amistad, la paz entre los países y fortalecer los derechos humanos. Considerando a la educación desde una dimensión teleológica, instrumento esencial en avalar la obediencia, respeto y solidaridad en todos los individuos.

En función al razonamiento, antes expuesto, la educación en derechos humanos, es el instrumento ideal para lograr una sociedad amante de la justicia. ¡Pero!, lamentablemente, aun en el siglo 20, la educación no ha sido el elemento principal de todos los países, parte de la Organización de las Naciones Unidas (ONU). Afirmación que, según el Foro Mundial para la Educación (UNESCO, 2000a): realizado en Dakar, capital de Senegal, ciudad ubicada en la Costa Atlántica de África, en la cual se efectuó el abordaje de la temática de la educación para todos y para reafirmar la "Declaración Mundial Sobre Educación para Todos y el Marco de Acción para Satisfacer las Necesidades Básicas de Aprendizaje" (pág. 1); aprobada en la Conferencia Mundial sobre Educación ejecutada en Jomtien, Tailandia de 5 al 9 de marzo de 1990. Según la cual todas las personas tienen el derecho de beneficiarse de una formación para satisfacer su necesidad educativa que contenga el aprendizaje del saber, hacer, vivir juntos y ser; con el fin de mejorar la sociedad en la cual se vive.

En tal sentido, Foro Mundial para la Educación (UNESCO, 2000b), afirmó que, a pesar de los avances relevantes de muchos países con relación a la educación, es inadmisible:

...que en el año 2000 más de 113 millones de niños no tengan 
acceso a la enseñanza básica, que todavía 880 millones de adultos sean analfabetos, que la discriminación por género siga dejando lastres en los sistemas educativos y que la calidad del aprendizaje, y la adquisición de valores humanos y de competencias estén lejos de atender las aspiraciones y las necesidades de los individuos y de las sociedades (pág. 1).

Del ejemplo anterior descrito, podemos decir que el punto de partida de lo expresado, es la negación del acceso al conocimiento para niños, niñas, adolescentes y adultos, para la integración activa en el desarrollo sustentable de la sociedad para fortalecer la cultura de paz y el equilibrio en cada uno de los países del mundo.

Por lo tanto, la praxis de la educación de los derechos humanos, es el medio idóneo para la participación efectiva de la sociedad y no solo visto desde la visión del acceso a la educación o de participar en una educación de calidad; se relaciona con el conocimiento real de la educación en derechos humanos desde el pragmatismo como corriente de pensamiento que considera como criterio de verdad lo que es útil y valioso; es decir, el derecho como norma jurídica creada para regir la vida en sociedad y los derechos humanos como prerrogativa creada para el bienestar del individuo y alcanzar en el mismo la autonomía del sujeto, optimizar la existencia del ciudadano, elevar su potencial en la integración de los procesos de la toma de decisiones en la conducción y formulación de políticas sociales, educativas, ambientales, culturales y económicas.

En el orden de las ideas, es primordial plantear una visión praxeológica de la educación de los derechos humanos y no solos desde el contenido teórico, sino sustentada en el respeto a la diversidad cultural, a la no violencia, el pluralismo político y la paz. La Asamblea General de las Organización de las Naciones Unidas (ONU), creen la importancia en capacitar a individuos en el componente del respeto humano, impulsando 
interacciones constantes, sólidas y armoniosas entre los integrantes de las comunidades en fortalecer y consolidar la interpretación recíproca, honesta y la paz. Desarrollando planes, programas y estrategias para la educación en derechos humanos; pero no han tenido difusión adecuada, obteniendo como resultado el agnosticismo de los ciudadanos.

De tal manera, que la Responsabilidades del Estado en materia de Derechos Humanos, juega un papel importantísimo que se rigen por los principios de: reconocerlos, respetarlos y garantizarlos, adecuar la normativa nacional, adoptar medidas para proteger los derechos, asegurar una adecuada reparación, sancionar a los culpables, organizar a los poderes públicos, recursos judiciales apropiados y eficaces, calificación de ilicitud para toda violación, deber de investigar, restablecer la situación jurídica o indemnizar, sancionar a los transgresores, adoptar medidas para que no se repita, difundir, impulsar la participación ciudadana, invocar y promover la responsabilidad social; la República Bolivariana de Venezuela, con la entrada en vigencia de la Constitución de 1999, es punta de lanza en materia de Derechos Humanos, teniendo la Carta Magna, más humanista y garantista del mundo.

Por consiguiente, en nuestra legislación interna, tenemos una institución especializada para garantizar las responsabilidades del Estado en Materia de Derechos Humanos. La Defensoría del Pueblo, quien representa una institución para el Poder Ciudadano en el desarrollo, cuidado de los Derechos Humanos y sus garantías establecidas en la Constitución de la República Bolivariana de Venezuela y los intereses legítimos, colectivos o difusos de los ciudadanos y ciudadanas según lo establecido en el artículo 280 de la Constitución de la República Bolivariana de Venezuela de año 1999. La visión de esta es: alcanzar la creencia y resguardo del bienestar de todos los individuos, específicamente los sujetos en escenarios de discriminación, pobreza y flaqueza. Todo esto, por medio de la integración participativa de las localidades, la transformación del Estado y la disminución del poder; 
impulsando una cultura acorde a los derechos de los ciudadanos, en la cual se encuentre cimentada en el respaldo internacional, la independencia de los pueblos y garantizar la plenitud de vida en toda la humanidad.

Según se ha citado, la Defensoría del Pueblo actúa cumpliendo una función pedagógica en relación con las ciudadanas y ciudadanos, así como las funcionarias y funcionarios públicos a fin de contribuir a consolidar los valores del bien común, la equidad social, la armonía y la autonomía, se constituye en intermediaria entre los individuos y la administración pública.

La Defensoría del Pueblo al identificar deficiencias, arbitrariedades, desviaciones de poder e incumplimiento de los deberes por parte de las funcionarias y funcionarios públicos, que lesionen los Derechos Humanos, persuade a las autoridades para que adopten medidas que rectifiquen y erradiquen tales conductas y recomienda soluciones; las autoridades, funcionarias y funcionarios públicos tienen el deber de cooperar con la labor defensoría y atender sus recomendaciones.

Dentro de sus atribuciones, atiende denuncias y orienta personas: toda persona que considere que sus derechos son afectados por alguna autoridad, entidad estatal o empresa de servicios públicos, puede presentar la denuncia en la Defensoría del Pueblo, la cual brinda orientación, investiga e intercede para encontrar una solución al problema, velar por la actuación en forma permanente.

La Defensoría del Pueblo exhorta a todas las instituciones públicas, locales, regionales y nacionales y a las instituciones que prestan servicios públicos, con la finalidad de verificar que actúen conforme a la ley y respetando los derechos de los ciudadanos y ciudadanas, visita e investiga las dependencias y establecimiento de los órganos del Estado, abarca cualquier órgano y funcionarios que se encuentra inmerso en el Poder Público: Nacional, Estadal o Municipal, en sus dependencias ejecutivas, Legislativas, Judiciales, Electorales, Militares y demás órganos del Poder Ciudadano, informa y 
recomienda; la Defensoría del Pueblo en función a los reclamos y las investigaciones iniciadas de oficio, presenta las observaciones $y$ recomendaciones para que las autoridades corrijan las conductas inconstitucionales, irregulares, discriminatorias, arbitrarias y se restituyan los derechos afectados, interpone recursos y acciones judiciales de inconstitucionalidad, auxilio, habeas corpus, habeas data, acciones de protección y demás acciones o recursos judiciales a que hubiere lugar, entre otras.

De lo antes expuesto, el punto inicio del estudio, relacionado con el agnosticismo en los ciudadanos en lo relativo a los Derechos Humanos, donde son manipulados continuamente por los medios de comunicaciones privados nacionales e internacionales, quienes crean matrices de opiniones sobre la continua violaciones de derechos humanos por parte de la República Bolivariana de Venezuela, tales como: "El Universal", domingo 8 de septiembre de 2013, entre los titulares sobre la denuncia de Venezuela del conocimiento de la Corte Interamericana de los Derechos Humanos fueron: "Con esta decisión Venezuela se convierte en un Estado forajido", "Venezuela se queda sola en la región con su resolución de retirarse de la Corte Interamericana"; otro ejemplo de la manipulación del medio de comunicación en mención, en la cual se elabore una matriz de ideas y opiniones contraria a la realidad, es la continuidad de la noticia, y sus mensajes subliminares.

De igual manera, como la noticia del jueves 12 de septiembre de 2013 a las 12:00 am titulares "Salida de Venezuela de Pacto de San José", "En efecto, la salida de Venezuela este tratado de protección de derechos humanos es grave" y los medios internacionales tales como CNN en Español, el 10 septiembre, 2013, dice entre sus titular: "Venezuela abandonó este martes formalmente el sistema interamericano de derechos humanos" o "EL NACIONAL", el 7 de abril de 2017 a las 07:04 pm, dice en sus titulares: "Venezuela en la "lista negra" de derechos humanos de CIDH", o el medio de 
comunicación internacional, "EL NUEVO HERALD", publicando el 16 de abril 2017 a las 12:20 pm, "Jefe de la OEA condena violación de derechos humanos a detenidos en Venezuela", entre otros y tratan de utilizar a los organismos internacionales, como organismos no gubernamentales (ONG), como por ejemplo, la ONG, Human Rights Watch, que en Mayo 5 de 2014, saco un comunicado diciendo "Castigados por Protestar", "Violaciones de derechos en las calles, centro de detención y el sistema de justicia en Venezuela"; todo con la finalidad, de crear una matriz de opinión ante la ciudadanía y la comunidad internacional, de que en Venezuela existe una continua infracción contra la vida humana y así lograr una intervención a nuestro país.

En tal sentido, se hace necesario un proceso de transformación curricular, especialmente en la instrucción de los derechos de las personas, en sistemas educativos en los preceptos del Estado venezolano, orientados a fortalecer los procesos educativos del individuo y de la sociedad, para impulsar una educación acorde con la realidad del país, que es un estado garante de los mismos, reconocido por la Organización de las Naciones Unidas (ONU) en septiembre del año 2016. En este contexto, la única forma de lograr concientizar al ciudadano en crear una visión objetiva con relación a los beneficios sociales de los sujetos, el consentimiento con lo establecida en la Constitución de la República Bolivariana de Venezuela y lograr esa transformación, en función de enaltecer la formación del ser humano para que no pueda ser víctima de manipulaciones a consecuencia de la ignorancia.

Por otra parte, son pocos los institutos de educación que poseen la materia de derechos humanos dentro de su malla curricular, dentro de las que podemos mencionar tres (03), la Universidad Bolivariana de Venezuela (UBV), la Escuela de Derechos Humanos de la Fundación "Juan Vives Suriá”, adscrita a la Defensoría del Pueblo y las Instituciones Educativas de las Fuerza Armada Nacional Bolivariana; en la carrera de derecho, de la diferentes universidades de Venezuela, no forma a los abogados en orientar, asesorar y guiar los 
derechos humanos, los planes de estudios no contempla dentro de su régimen semestral de formación, la materia de Derechos Humanos y la que la posee la tienen como una asignatura optativa; lo que implica que ni los abogados, profesionales del conocimiento técnico jurídico, son formados con una conciencia humanista que le permita promover la justicia social de las realidades sociales de nuestro país y su constitución.

Por ello, es necesario la formación del docente y las mallas curriculares de las instituciones educativas, el mejoramiento de la calidad en la instrucción de los Derechos Humanos, para romper con los viejos estereotipos, de la enseñanza de los derechos humanos, para iluminar la iniciativa de innovación didáctica junto a la esencia de un nuevo sentir y actuar de los docentes de adultos. Consecuentemente, las instituciones asumen, que el docente posee los argumentos epistemológicos y contextuales que rodean la práctica de la enseñanza, capaces de generar y conducir cambios en el conocimiento por medio de la difusión del saber, la cultura, y la formación profesional con conciencia nacional.

Ahora bien, dentro de la malla curricular de la Universidad Bolivariana de Venezuela y los Institutos Educativos de la Fuerza Armada Nacional Bolivariana, que contempla la materia de derechos humanos, solo vislumbra la unidad curricular por un solo periodo, en la (UBV), en el programa de formación de Estudios Jurídicos en el trayecto 3, en los tramos 1 y 2, y en las Instituciones Militares también se ve por un solo periodo.

A nivel de las Escuelas Militares, en todas las mallas curriculares de la misma, se concibe la materia de los derechos humanos, generalmente se ve durante el primer periodo de formación y se fortalece con charlas y talleres antes de graduarse y durante la vida profesional se refuerza en ocasiones. En la Escuela de Formación de Guardias Nacionales "G/D "Víctor Anselmo Fernández Escobar", el diseño curricular del programa de formación de la tropa profesional de la Fuerza Armada Nacional Bolivariana "Teniente Pedro 
Camejo", contempla el adiestramiento de los derechos humanos en las fases de formación, pero se observa en las charlas o talleres que se dictan antes de graduarse los alumnos, no tener un conocimiento adecuado sobre los derechos humanos. De allí, se plantea como objetivo general analizar la evolución histórica de los derechos humanos en la República Bolivariana de Venezuela, con el propósito de conocer el porqué del agnosticismo pedagógico de los derechos humanos en la ciudadanía.

\section{Consideraciones Teóricas}

De conformidad con la estructura gnoseológica, asumida en la presente artículo, para profundizar desde la perspectiva teleológica del otorgamiento al ser humano de derechos, desde una perspectiva teórica en la búsqueda de la justicia material, como un proceso en construcción del conocimiento, que permita el entendimiento real de los derechos humanos en la República Bolivariana de Venezuela y así lograr el respecto de los mismos, basado en los axiomas de los Derechos Humanos; la enseñanza, como sinónimo de formación y el derecho, como norma jurídica creadas para regir la vida en sociedad y el teorema descrito en el contexto empírico: DERECHO más (+) CONOCIMIENTO, es igual (=) a: "RESPETO".

\subsection{Base Filosófica de los Derechos Humanos}

Es importante acotar que, el origen de los derechos surge en Grecia, es donde el pensamiento del hombre se va más allá, de la naturaleza animal y junto a la filosofía, es la expresión de lo apolíneo-dionisíaco, desde la organización filosófica de la voluntad de poder y de la vida, que sirve de base a la corriente humanista. Ahora bien, para comprender esta realidad histórica y los cambios que se han suscitado en la evolución del tiempo; para hablar de Derechos Humanos, se habla de dos corrientes que han luchado por el reconocimiento de las garantías del hombre desde el punto de vista del 
pensamiento jurídico; la CORRIENTE "IUSNATURALISTA" y la corriente "IUSPOSITIVISTA", cada corriente ve los derechos humanos desde su punto de vista, creando su propia teoría, de conformidad con sus propios paradigmas, motivo por el cual no podemos hablar de verdades absolutas, dice Orozco y González (s.f.):

...del estudio de la filosofía jurídica encontramos diversas formas de explicar la fenomenología del derecho a través de las variadas corrientes ius epistemológicas que han ido desarrollándose a lo largo de la historia de esta ciencia: lus naturalismo, lus positivismo, racionalismo, o lus realismo son algunas de ellas, por mencionarlas tan sólo en forma ilustrativa (pág. 2).

Lo que si podemos afirmar, todas son producto de la evolución histórica del hombre en la tierra, desde el mismo nacimiento de su existencia. De allí que, al referirnos a la corriente del IUSNATURALISMO, son los derechos provenidos de la naturaleza humana, que afirman la existencia de estos, desde el mismo nacimiento del ser humano.

Por su parte, la corriente IUSPOSITIVISTA, afirma la existencia del derecho, a partir de su fundamentación plasmada en ley, por lo tanto, si un derecho no es reconocido y garantizado por ley, ese derecho es ilegitimo; así mismo, existen dos tipos de corriente positivista, las cuales se especifican como positivismo radical y positivismo moderado. Señalan Orozco y González (s.f.): "el positivismo radical sostiene que el derecho natural es sólo una simple ideología que puede debilitar o fortalecer al derecho positivo; en cambio, el positivismo moderado considera que el término derecho debe ser empleado exclusivamente para el derecho positivo..." (pág. 2). Esto significa que cada corriente de pensamiento, cimenta su propia teoría conforme a sus diferentes enfoques, por consiguiente, no hay un conocimiento real y se crean una diversidad de información entorno a los Derechos Humanos, agenciando el desconocimiento de los mismos. 
En referencia a lo dicho y precisando de una vez, en razón a las dos corrientes descrita, se afirma que siempre ha existido un conflicto entre las misma, buscando justificar cada una sus teorías, independiente que haya una jerarquía jurídica de orden superior como garantía humana o contrariamente al naturalismo, que para que el derecho pueda ser reconocido tiene que ser pronunciadas por los órganos competentes, elaborada en consentimiento con los procesos determinados, por los legisladores del gobierno que ostenta el poder.

En ese mismo sentido, es a partir de la época griega, donde las concepciones filosófica de la época, tenía como eje principal de estudio la persona, de donde se desprenden diferentes juicios, con atributos esenciales para el desarrollo de políticas de estados, dirigida a la organización, protección y felicidad individual de sus pueblos; la más destaca fue la Estoica, de donde nace el pensamiento filosófico, del hombre sabio y de las leyes naturales, según Solís (2013), "Evolución de los Derechos Humanos", la misma manifiesta:

...la filosofía estoica abrió nuevas perspectivas al desarrollo humano. El hombre no fue ya al estrecho ciudadano de la polis, sino el miembro de una comunidad universal. Además, se acentuó, la idea de la dignidad, de que todo lo que tiene rostro humano tiene valor natural de libertad y de igualdad (pág. 79).

En el orden de las ideas anteriores, los filósofos estoicos hacen alusión al desarrollo humano, donde todos los hombres son iguales en derecho, partiendo de las leyes naturales en armonía con la razón. Es evidente entonces, para los escritores estoicos, la naturaleza humana del hombre parte de la igualdad como seres racionales y en tal sentido, disfrutan de los mismos derechos y deberes.

Ahora bien, Roma es otro de los grandes referentes de la antigüedad, abarca uno de los periodos más largos de historia comprendido entre los siglos 
siglo VIII, antes de Cristo (a.C.) y finales del siglo V, después de Cristo (d.C.). Su mayor mérito fue el desarrollo de un amplio cuerpo de leyes, tomando los logros de las civilizaciones pasadas y obteniendo poder absoluto en base a la Ley.

\subsection{Base filosófica de los Derechos Humanos desde las constituciones existentes en Venezuela}

En la República de Venezuela, se fueron desarrollando diferentes constituciones y reformas a las mismas, para reconocer derechos, tales como: regreso de exiliados políticos, liberación de presos políticos, restitución de garantías constitucionales, creación de partidos políticos, disfrute de las libertades civiles, se crean organismos gubernamentales y leyes ordinarias para garantizar las políticas públicas de Estado (Contraloría General de la Republica, el Banco Central, el Ministerio de Sanidad, ley de Hidrocarburos, ley de Universidades), se funda Fedecámaras, se realizan elecciones directas, secretas y universales, se incorpora a la mujer en la política, se impulsa la construcción de obras públicas, se firma el "Pacto de Punto Fijo" se dicta la "Declaración de principios y programas mínimo de Gobierno".

Igualmente, la negación de derechos tales como: la ilegalidad de las Organizaciones de izquierda y expulsión de dirigentes políticos en el año de 1937, persecuciones políticas y torturas. Durante el período antes nombrado, Desde el año 1935 al año 1958, en la República de Venezuela existieron 4 constituciones, que serán tomadas en referencia para dar a conocer, el desenvolvimiento, la evolución de los derechos de los venezolanos.

La primera carta magna del periodo llamado, "Transición a la Democracia" fue, la Constitución de los Estados Unidos de Venezuela, de fecha 20 de Julio de 1936 , año $127^{\circ}$ de la Independencia y $78^{\circ}$ de la Federación, conformada por IX Títulos y cada Título por Secciones, para un total de 134 artículos. En el Titulo II, se contemplaban los derechos y deberes 
de los venezolanos y especifica que "La Nación Garantiza a los Venezolanos", específicamente el respeto a la vida, liberación individual y con garantía al debido proceso; la libertad de tránsito; la propiedad; el trabajo; la inviolabilidad de la correspondencias; la inviolabilidad del hogar; la abolición del reclutamiento forzoso para el servicio de las armas; la esclavitud y garantizándole a cualquier esclavo que pise el territorio venezolano ese derecho; la libertad de petición y de acusar ante los tribunales a los funcionarios públicos; el derecho de sufragio, solo para el hombre mayor de 21 años; la liberación de enseñanza; la libertad de religión; la libertad de reuniones sin armas; el rescate de pensamiento, sujetas a restricciones penales (La doctrina comunista y anarquista, fueron consideradas contrarias a la independencia, a la política, a la paz social y eran considerados traidores a la Patria).

La segunda constitución, fue la Constitución de los Estados Unidos de Venezuela de 1945, Constituida por 140 artículos, del periodo presidencial del presidente Isaías Medina Angarita, entre los derechos y deberes de los venezolanos tenemos: la nacionalidad se tiene por nacimiento y por naturalización; la inviolabilidad de la vida, de la propiedad, de la correspondencia, la libertad personal; la liberta de transitar, vacaciones anuales y remuneradas; libertad de petición, religiosa, de enseñar; el derecho de sufragio. Se restituye el derecho de libertad de pensamiento (La doctrina comunista ya no es un delito) y se le da el derecho al sufragio a la mujer.

La tercera constitución, de transición a la democracia, es la Constitución de los Estados Unidos de Venezuela de 1947, Constituida por 253 artículos, a diferencia de las dos anteriores, donde el Titulo II, contemplaban los derechos y deberes de los venezolanos. Esta constitución contenía en el Titulo III, los deberes, derechos individuales y sociales de los venezolanos. Dando una ampliación mayor de derechos a los ciudadanos, divididos en capítulos. El Capítulo I, las Disposiciones Generales, del artículo 20 al 28; el Capítulo II, 
las Garantías Individuales, del artículo 29 al 46; el Capítulo III, de la familia, del artículo 47 al 50; el Capítulo IV, de la Salud y de la Seguridad Social, artículo 51 y 52; el Capítulo V, de la Educación, del artículo 53 al 60, dándole carácter constitucional y promoviendo la privatización de la educación; el Capítulo Vl; del trabajo, del artículo 61 al 64; el Capítulo VII, de la Economía Nacional, del artículo 65 al 75; el Capítulo VIII, de la Suspensión y Restricción de Garantías, del artículo 76 al 78; en el Título IV, de la Soberanía y del Poder Público, dedicando el Capítulo I, al Sufragio, desde el artículo 79 al 83; el Capítulo II del Poder Público y su Ejercicio, del artículo 84 al 92; el Capítulo III, de las Fuerzas Armadas Nacionales, del artículo 93 al 103; el Capítulo IV, de las Relaciones Internacionales, del artículo 104 al 108; los siguientes Títulos y Capítulos van a tratar sobre el Poder de los Estados y su División Política y la formación de las Leyes.

La cuarta y última constitución del periodo denominado transición a la democracia, fue la constitución de 1953, promulgada por el presidente constitucional, Marcos Pérez Jiménez. La nación venezolana deja de llamarse con el nombre de "Estados Unidos de Venezuela", para llamarse "República de Venezuela", regida por los principios de Gobierno Federal, Democrático, Electivo, Representativo, Responsable, Alternativo, Independiente y Libre de toda Dominación o Protección Extranjera. La constitución está constituida por 142 artículos. El título III, dedicado a los Deberes y Derechos Individuales y Sociales de los venezolanos, comenzando con el artículo 28, que establecía: "Todos los venezolanos tienen el derecho de hacer lo que no perjudique a otro y nadie está obligado a hacer lo que la ley no ordene ni impedido ejecutar lo que ella no prohíba." Y culmina con el artículo 32; el Capítulo II, consagrado a los Deberes de los Venezolanos y de los Extranjeros, vislumbrado en los artículo 33, que instauraba defender a la patria, el servicio militar obligatorio, obedecer la Constitución y las Leyes; y el artículo 34 los deberes de los extranjeros; el Capítulo III, sobre las Garantías Individuales, desde el artículo 
35, sobre las garantías de los venezolanos, enunciadas en 13 numerales y los artículos 36 y 37 la restricción o suspensión de las garantías ciudadanas; los Títulos IV, V y VI, hablaban sobre la Soberanía y del Poder Público, de los Poderes Públicos Nacionales y de la Reforma de la Constitución. He de observarse, que esta Constitución, restringió una gran cantidad de derechos que contemplaba la Constitución de 1947.

Con referencia a lo anterior, el 16 de enero de 1961, se realiza la nueva Constitución de la República de Venezuela, tomando la constitución de 1947, constituida por XII Títulos y 252 artículos. El Título III, establece los Deberes, Derechos y Garantías de los habitantes de la República de Venezuela; el Capítulo I, instaura las disposiciones generales, que van desde el artículo 43 al 50; el capítulo II, asienta los deberes, desde el artículo 51 al 57; el capítulo III, especifica los derechos individuales, desde el artículo 58 al 71; el capítulo IV, detalla los derechos sociales, desde el artículo 72 al 94; el capítulo V, enumera los derechos económicos, desde el artículo 95 al 109; el capítulo VI, enuncia los derechos políticos, desde el artículo 110 al 116. Resulta oportuno realizar la siguiente observación, es a partir de Constitución de 1961, cuando se puede hablar de Derechos Humanos y Derechos Fundamentales en nuestro país. A razón de la Declaración Universal de los Derechos Humanos de 1948 y los Pactos de los Derechos Sociales, Económicos, Culturales, Civiles y Políticos de 1966.

Es evidente entonces, que desde la promulgación de esta constitución del 61 y la Declaración Universal de los Derechos Humanos del 10 de Diciembre de 1948, por la Organización de las Naciones Unidas (ONU), proclamada en la Asamblea General de las Naciones Unidas, según resolución 217 A (III), como una idea común para todos los países miembros; es para ese entonces, que en la República de Venezuela, comienza la era de los Derechos Humanos, con la salvedad, que solo se hace referencia a los Derechos Humanos, desde el punto de vista dogmático, hasta el año de 
1978; el dogmatismo se concibe como una teoría que sirve para sustentar principios, fundamento de lo es el deber ser, lo que es indiscutible.

\section{Metodología}

Esta investigación es de tipo documental descriptiva, el método a emplear para la obtención de las posibles respuestas al objetivo planteado es el método cualitativo etnográfico, la observación participante y la revisión bibliográfica. En tal sentido, el carácter cualitativo busca profundizar en la investigación, planteando un diseño abierto y emergente desde la representación etnográfica, estudiando no solo al sujeto, sino el entorno y la contextualización del ambiente en que se desarrolla el mismo, utilizando como técnica principal de recolección de los datos, la observación participativa en el escenario educativo planteado, la Escuela de Formación de Guardias Nacionales "Gral. Div. (F) Victo Anselmo Fernández Escobar". En lo marcado en las observaciones anteriores, el investigador cualitativo, según Angrosino (2012a), expone:

Se interesan en acceder a la experiencia, interacciones y documentos del material a estudiar, se abstienes de establecer al principio, conceptos claros del objeto que se estudia, parte del principio de que los métodos y las teorías deben ser propias para lo que se estudia, el investigador es parte fundamental en la investigación, desde el punto de vista de su propia experiencia en relación al objeto de estudio, las notas de campo, la transcripción, descripción e interpretación de los hallazgos (pág. 11).

Es evidente entonces, que el método cualitativo se refiere a las vías empleadas en la apropiación y generación y/o construcción del conocimiento. En esta misma dirección, desde la priori etnográfica, implica la recogida de información, con énfasis en las relaciones sociales, las creencias y los valores de una sociedad, dice Angrosino (2012b): "el producto etnográfico es un informe que incorpora la información recogida por el método etnográfico en 
una descripción integral de la cultura de la comunidad" (pág. 11).

De lo anterior citado, se puede decir, que los datos obtenidos fueron recogidos de la revisión bibliográfica sobre los derechos humanos y de la observación realizada durante la formación de las promociones 104, 105, 106 y 107, egresadas de la Escuela de Formación de Guardias Nacionales "Gral. Div. (F) Victo Anselmo Fernández Escobar"; siendo el investigador, plaza de la institución educativa en mención e instructor de la materia de derechos humanos; realizándose una descripción detallada de las bases filosóficas de los derechos humanos en la República Bolivariana de Venezuela.

\section{Conclusiones}

Existe en la República Bolivariana un agnosticismo sobre los derechos humanos, basado en la falta de políticas públicas orientadas a la enseñanza de los mismos a nivel educacional; producto de la falta de una formación continua pedagógica en los institutos educativos de la educación andragógica, porque no vislumbran los componentes esenciales de derechos humanos en las mallas curriculares y los que se contempla en los mismos, sólo lo ven como una materia opcional o durante el primer periodo de formación, lo que trae como consecuencia el desconocimiento real de que son los derechos humanos y por ende comprender, interpretar y analizar las garantías del bienestar social, económico, educativo y político.

\section{Referencias}

ACNUDH (2012). ¿Qué son los derechos humanos? Ginebra, Suiza: La Oficina del Alto Comisionado para los Derechos Humanos. Recuperado de: https://www.ohchr.org/sp/issues/Pages/WhatareHumanRights.aspx Angrosino, M. (2012a,b). Etnografía y observación participante en Investigación Cualitativa. Mejía Lequerica, Madrid, España: Ediciones Morata, S. L. 
Orozco, I., \& González, J. (s.f.). Los derechos humanos desde una perspectiva tridimensional. México: Universidad Interamericana para el Desarrollo, UNID.

Solís, B. (2013). Evolución de los Derechos Humanos. México: Biblioteca jurídica virtual del Instituto de Investigaciones Jurídicas de la UNAM. Recuperado de:

https://archivos.juridicas.unam.mx/www/bjv/libros/7/3100/9.pdf

UNESCO $(2000 a, b)$. Marco de Acción de Dakar. Foro Mundial para la Educación. Senegal, Costa Atlántica de África: La Organización de las Naciones Unidas para la Educación, la Ciencia y la Cultura. 


\section{Richard Manuel Salazar Brito \\ e-mail: raam2623@hotmail.com}

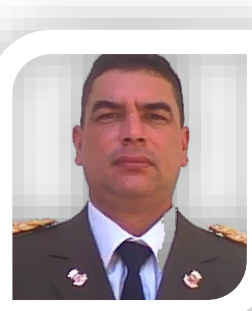

Nacido en Caracas, distrito capital, República Bolivariana de Venezuela. Licenciado en Ciencias y Artes Militares: Academia Militar de la Guardia Nacional Bolivariana, Año 1993; Especialista en Seguridad: Escuela Superior de Defensa Militar y Orden Interno Caracas, año 2001; Diplomado en Derecho Internacional de los Refugiados: Universidad Católica del Táchira, año 2013; Abogado por la Universidad Bolivariana de Venezuela, San Cristóbal, año 2015; Magíster en Seguridad y Defensa de la Nación, Instituto de Alto Estudio de la Defensa Nacional "Gran Mariscal de Ayacucho Antonio José de Sucre", año 2015; Diplomado en Derechos Humanos y Estado Social: Escuela Nacional de la Magistratura, año 2017.

El contenido de este manuscrito se difunde bajo una Licencia de Creative Commons ReconocimientoNoComercial-Compartirlgual 4.0 Internacional 